\title{
Prosthetic Hip Joint Infection: An Aluminium Mold for Intraoperative Production of Antibiotic-loaded Cement Hip Prostheses: 3 Cases Report
}

Truong Nguyen Khanh Hung ${ }^{1 *}$, Tran Binh Duong ${ }^{1}$, Tran Phuoc Binh ${ }^{1}$, Dao Thanh $\mathrm{Tu}^{1}$, Le Nghi Thanh Nhan ${ }^{2}$, Cao Thi ${ }^{3}$ and Le Van Tuan ${ }^{1}$

${ }^{1}$ Orthopedic and Trauma Department, ChoRay Hospital, Ho Chi Minh City, Vietnam

2Orthopedic Department, Hue Medical University Hospital, Hue City, Vietnam

${ }^{3}$ Orthopedic and Rehabilitation Department, Ho Chi Minh Medical and Pharmacy University, Ho Chi Minh City, Vietnam

*Corresponding author: Truong Nguyen Khanh Hung, Orthopedic and Trauma Department, ChoRay Hospital, Ho Chi Minh City, Vietnam, Tel: +84942888811; E-mail: drhung.bvcr@gmail.com

Received Date: Mar 12, 2018; Accepted Date: Mar 21, 2018; Published Date: Mar 29, 2018

Copyright: ( 2018 Hung TNK, et al. This is an open-access article distributed under the terms of the Creative Commons Attribution License, which permits unrestricted use, distribution, and reproduction in any medium, provided the original author and source are credited.

\section{Abstract}

Introduction: To treat hip prosthetic infection, 2-stage revision, including removal and reimplantation, remains the standard treatment for prosthetic infection. Articulating cement spacer has been shown to provide better functional results after reimplantation. However, its cost as a manufactured product is not cheap and the choice of antibiotics is not flexible either. We designed an aluminium mold to make an antibiotic impregnated cement spacer, and replaced it between the first and the second stage. The current study was conducted to test their clinical efficacy.

Case report: We report 3 cases of hip prosthetic infection treatment by using antibiotic impregnated cement spacer made by an aluminium mold. All patients presented to us in first 2-years postoperation and all had a deep infection. Three patients with infected total hip arthroplasties were treated with 2-stage revision using articulating spacers made by an aluminium mold and had a good result.

Conclusion: Treating hip prosthetic infection with these articulating spacers eradicates infection effectively, improves the life quality before reimplantation and provides good final results without significant complications.

\section{Introduction}

Periprosthetic joint infection (PJI) is a truly devastating complication of total joint arthroplasty [1]. It adversely impacts the patient, by causing functional disability, increased morbidity and also mortality [2]. The management of PJI currently is far from optimal, often resulting in the need for prolonged hospitalization, administration of long term intravenous antibiotics, and the need for multiple surgical interventions [3]. The protracted course of treatment results in a massive financial burden on the treating institution and the health system on a national level. The incidence of PJI has been increasing steadily over the last decade, both in terms of the absolute number of cases, as well as the proportion of primary total hip and knee arthroplasties that succumb to infection $[3,4]$. The resistance profile of infecting organisms has also changed over the recent years with an increase in the number of surgical site infections and PJIs being caused by antibiotic resistant organisms [5,6]. While recurrence of PJI after treatment is not common, eradication rates as low as $16-37 \%$ has been shown with infection of certain organisms treated with less-aggressive strategies $[7,8]$. The extensive treatment required to appropriately treat a patient with PJI is significantly more expensive than that for aseptic loosening after primary total joint arthroplasty [3], and treating institutions are experiencing a decline in reimbursement along with the development of penalties for infection associated readmission.

\section{Construction of the spacer mold}

The mold was produced in a computerized CNC machine (DATRON Booth N-6021) and consists of 2 parts. It is made of high strength aluminium (AA7075 T6 3.4365; density $2.8 \mathrm{~kg} / \mathrm{dm}^{3}$ and tensile strength $520560 \mathrm{Nm} / \mathrm{mm}^{2}$ ) and coated by ANODE technique. Half of the coating penetrates into the base material, which results in an increase of $25 \mathrm{~m}$, leading to a standard coating thickness of $50 \mathrm{~m}$. The mold can be cleaned and sterilized for reuse (Figure 1).

\section{Case Report}

Informed consent to publish the case report was obtained from the patients.

\section{Case 1}

The patient is a 59-year-old Vietnamese man. This patient has diabetes type 2 for 10 years; he was still on treatment with glucose level in control. In 2011, in the absence of previous traumas, he was diagnosed with bilateral femoral head avascular necrosis. Pain-killer treatment, corticosteroid therapy and nonsteroidal anti-infammatory drugs were performed in another medical Centre, in other Vietnamese region. Because of poor clinical improvement, he also underwent left hip arthroplasty. In 2013, he was operated with the right hip. 1 year after he developed PJI of the left hip with symptoms such as pain, swelling local, warmth and mild fever. He was hospitalized and debrided for 3 months. He was discharged after that and antibiotics treatment. On July 2016, he was in lot of pain on the same area with an abscess $3-5 \mathrm{~cm}$. He was debrided in hospital X and transferred to Cho 
Citation: Hung TNK, Duong TB, Tu DT, Nhan LNT, Thi C, et al. (2018) Prosthetic Hip Joint Infection: An Aluminium Mold for Intraoperative Production of Antibiotic-loaded Cement Hip Prostheses: 3 Cases Report. Med Rep Case Stud 3: 153. doi:10.4172/2572-5130.1000153

Page 2 of 5

Ray hospital after 1 month in the unimproved infectious condition. He underwent debrided, serological tests, synovial fluid tests, antibiogramme and VAC drainage.
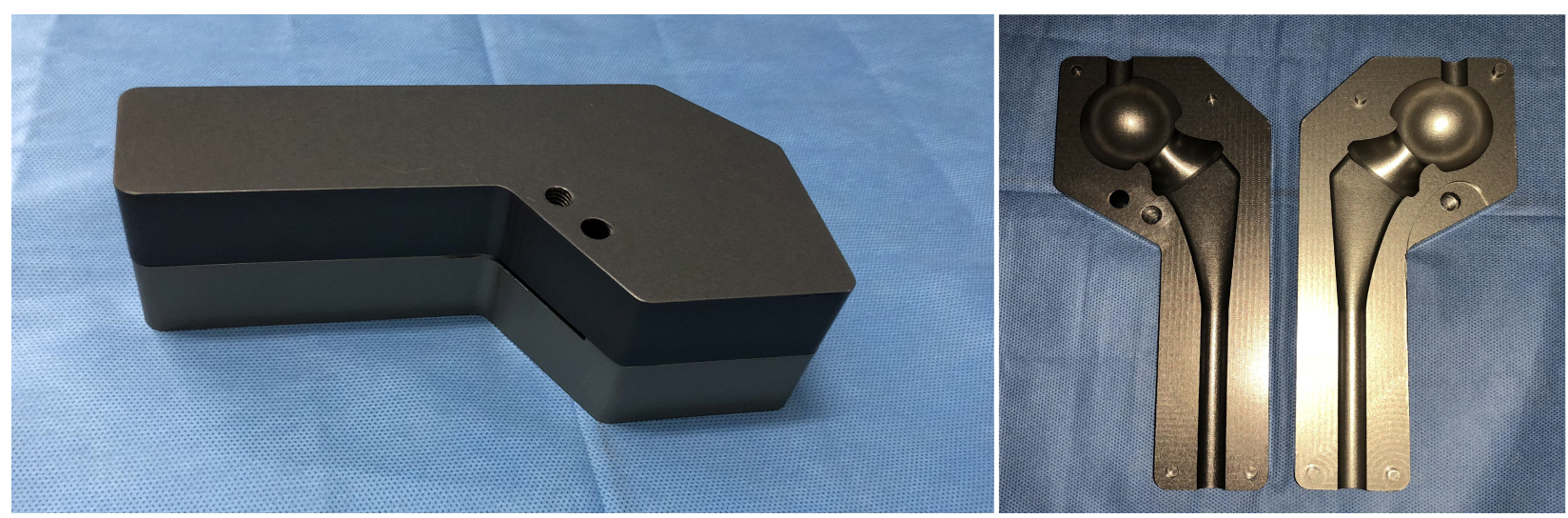

Figure 1: Aluminium mold.

After 2 time debridement and VAC drainage, he was impregnated with antibiotic-loaded cement spacer, which was made by aluminium mold and loaded Teicoplanin 1,2 g, and Vancomycin $2 \mathrm{~g}$ (Synovial fluid tests result: Enterococcus Faecium, multiple antibiotic resistance). He was discharged after 2 weeks with the infection in control, dry wound. He keeps on using oral antibiotic treatment in 6 weeks (Figure $2 \mathrm{a}$ and $2 \mathrm{~b})$.

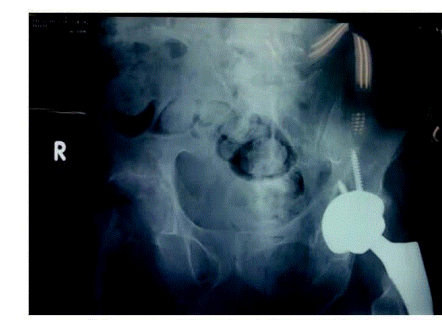

Figure 2a: Xray

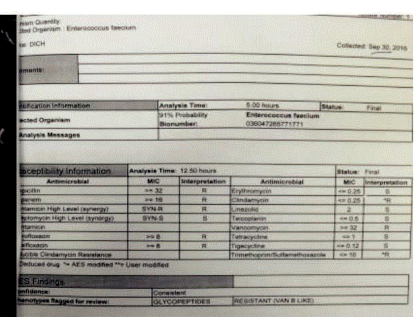

Figure 2b: antibiogramme
Figure 2: (a) Xray (b) Antibiogramme.
After 1 month, he checked up in Cho Ray hospital, his wound was healed, left leg function was good, and infectious condition was improved. Serological Tests: RBC 4.58 T/L, WBC: $6.66 \mathrm{~T} / \mathrm{L}$ (Neu: 58.9\%), CRP: 56 mg/L, Vs: 1 h 39 mm, 2 h 58 mm, Procalcitonin: 0.134 $\mathrm{ng} / \mathrm{ml}$ (Figure 3).

3 months after the end of treatment, the patient is totally asymptomatic, the spacer is stable and he reports an good function, and his Serological Tests results: RBC 5.48 T/L, WBC: 6.89 T/L (Neu: 53.9\%), CRP: $17 \mathrm{mg} / \mathrm{L}$, Vs: $1 \mathrm{~h} 16 \mathrm{~mm}, 2 \mathrm{~h} 27 \mathrm{~mm}$ (Figure 4). After 6 months, he also underwent left hip revision arthroplasty safely.

\section{Case 2}

The patient is a 50-year-old Vietnamese man. In 2009, in the absence of previous traumas, he was diagnosed with left femoral head avascular necrosis. Pain-killer treatment, corticosteroid therapy and non-steroidal anti-inflammatory drugs were performed in another hospital. Because of poor clinical improvement, he also underwent left hip arthroplasty in 2010. 1 year after he developed PJI of the left hip with symptoms, such as pain, swelling local and warmth. Antibiotic treatment and nonsteroidal anti-inflammatory drugs were performed.

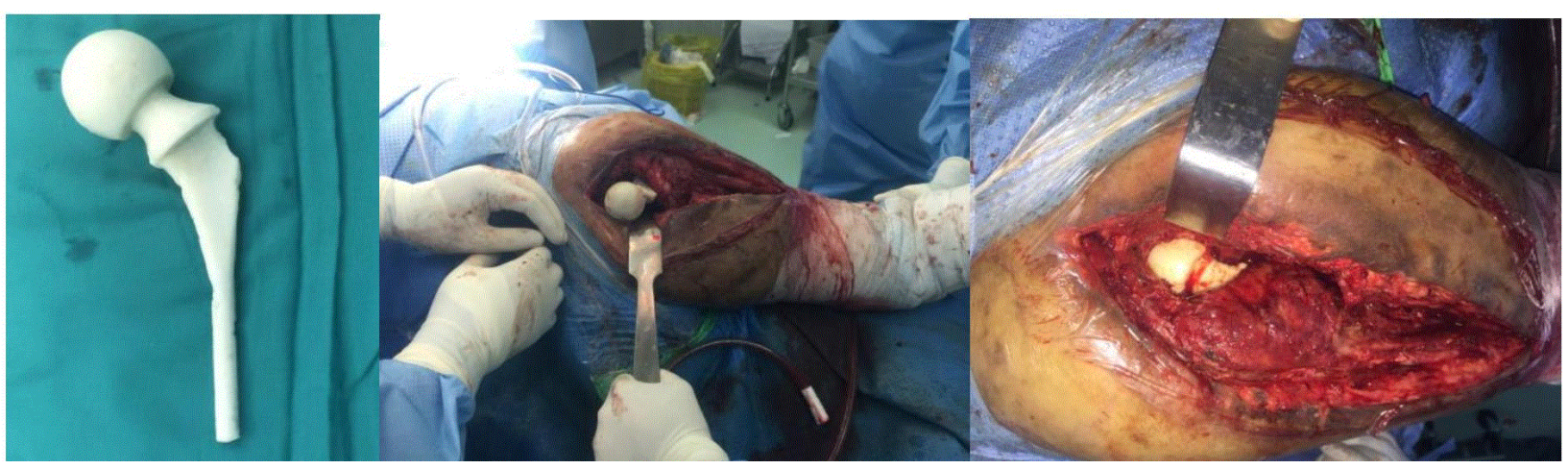

Figure 3: Spacer. 
Citation: Hung TNK, Duong TB, Tu DT, Nhan LNT, Thi C, et al. (2018) Prosthetic Hip Joint Infection: An Aluminium Mold for Intraoperative Production of Antibiotic-loaded Cement Hip Prostheses: 3 Cases Report. Med Rep Case Stud 3: 153. doi:10.4172/2572-5130.1000153

Page 3 of 5

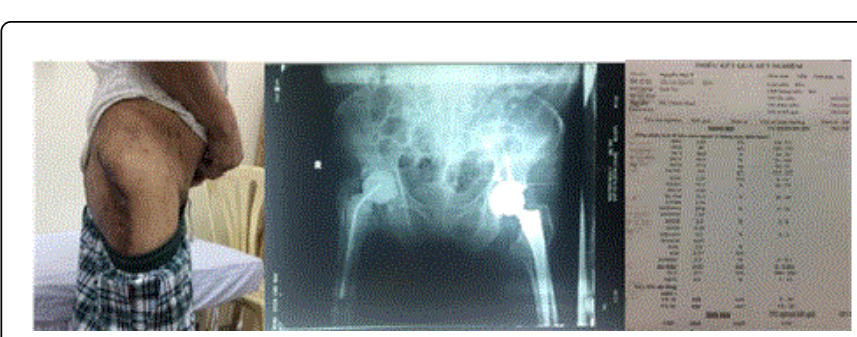

Figure 4: After 3 months.

On February 2017, his clinical improvement was too bad with pus from drainage incision. He has been admitted to that hospital, and underwent debrided, VAC in three times. On May 2017 he underwent debrided again, removed the prosthesis, used PROSTALAC (made by hand) and then transferred to Cho Ray hospital in the unimproved infectious condition. At Cho Ray hospital, he underwent debrided to remove the PROSTALAC, serological tests, synovial fluid tests, antibiogramme and VAC drainage. After 3 times debridement and VAC drainage, he was impregnated with antibiotic-loaded cement spacer, which was made by aluminium mold and loaded: Imipenem 2 $\mathrm{g}$, and Vancomycin $2 \mathrm{~g}$ (Synovial fluid tests result: Staphy-lococcus aureus, multiple antibiotic resistance). He was discharged after 2 weeks with the infection in control, dry wound. He keeps on using oral antibiotic treatment in 6 weeks (Figure 5).

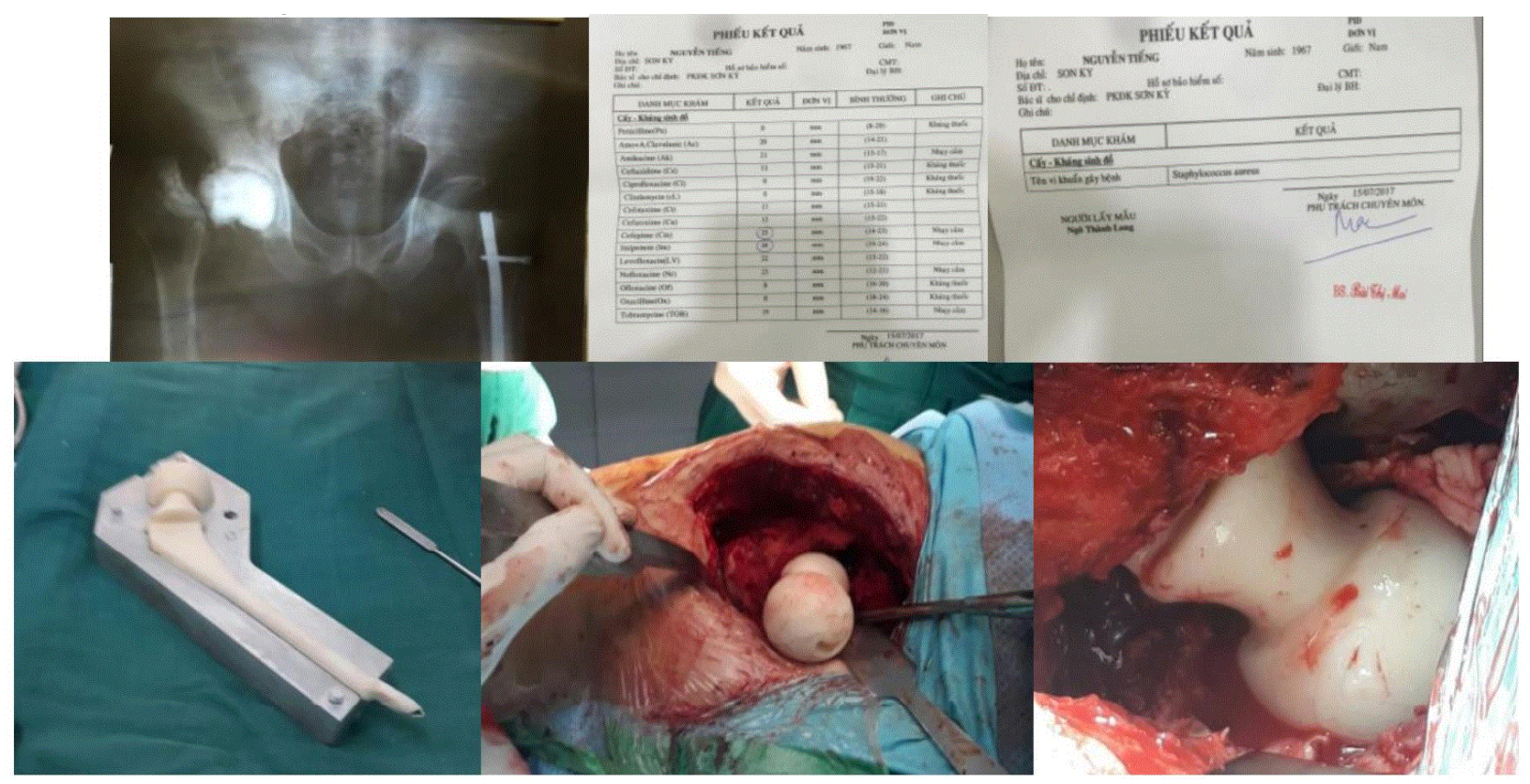

Figure 5: Antibiotic-loaded cement spacer.

Three weeks post operation, his wound was healing, and his serological tests results: WBC normal, ESR $1 \mathrm{~h}: 58 \mathrm{~mm}, 2 \mathrm{~h}: 84 \mathrm{~mm}$, CRP: $34,2 \mathrm{mg} / \mathrm{l}$. After 3 months, he checked up in Cho Ray hospital, his wound was healed, left leg function was good, and infectious condition was improved. Serological Tests: WBC: normal, ESR: normal, CRP: 5,5 mg/L (Figure 6).

He also underwent left hip revision arthroplasty after 7 months.

\section{Case 3}

The patient is a 50 year old Vietnamese man. In 2015, this patient suffered from traffic accident (hit by train), then he admitted to hospital X with diagnosis: multi trauma; left renal injury grade II, epidural hematoma of left brain, fracture of the posterior wall of the left acetabulum, fracture of the left femoral head. He underwent 10 weeks in ICU. Because of good clinical improvement, he was transferred to orthopedic department. Then, he was operated for total hip replacement. After 3 weeks post-operation, patient has infection after hip replacement; at hospital X he was debrided with VAC (13 times). On January 2017, His infection are not reduced, patient was removed all component, used PROSTALAC (made by hand). After 1 month, the infection was not reduced, and then he was transferred to Cho Ray hospital.

At Cho Ray hospital, he underwent debridement 3 times and VAC drainage, and replaced an intra-operative production of antibioticloaded cement spacer, which combined 2 packs cement 40 g, 1,2 g Teicoplanin and $2 \mathrm{~g}$ Vancomycin (Synovial fluid tests result: Staphylococcus aureus, MRSA). After 2 weeks, he was discharged with healing wound. He also kept on using oral antibiotic treatment in 6 weeks (Figure 7). 
Citation: Hung TNK, Duong TB, Tu DT, Nhan LNT, Thi C, et al. (2018) Prosthetic Hip Joint Infection: An Aluminium Mold for Intraoperative Production of Antibiotic-loaded Cement Hip Prostheses: 3 Cases Report. Med Rep Case Stud 3: 153. doi:10.4172/2572-5130.1000153

Page 4 of 5

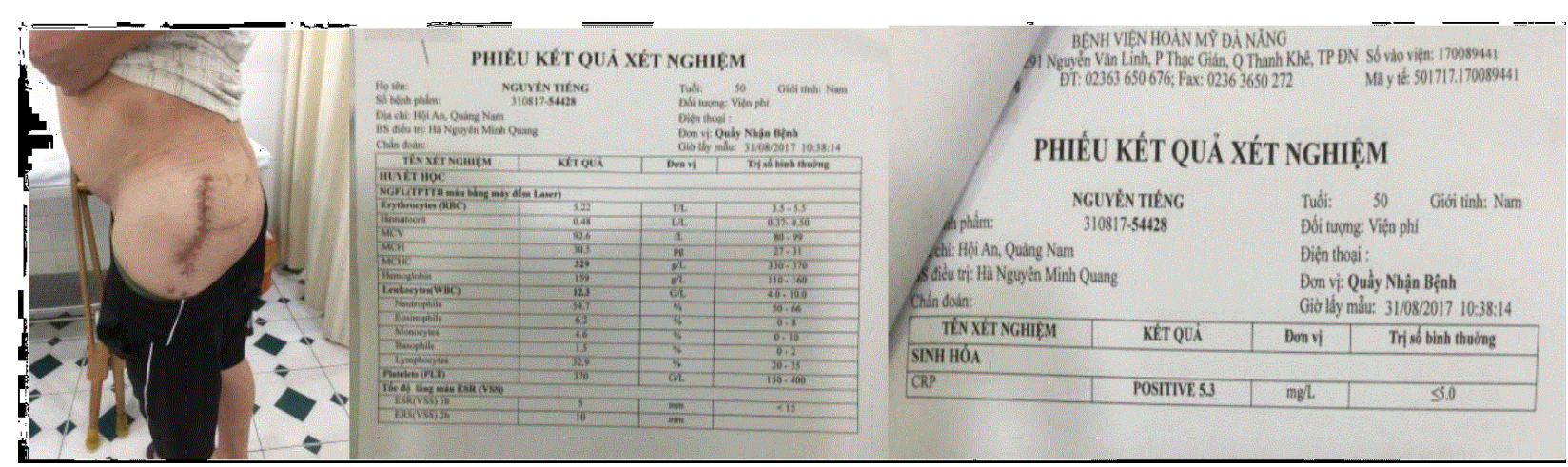

Figure 6: After 3 months.
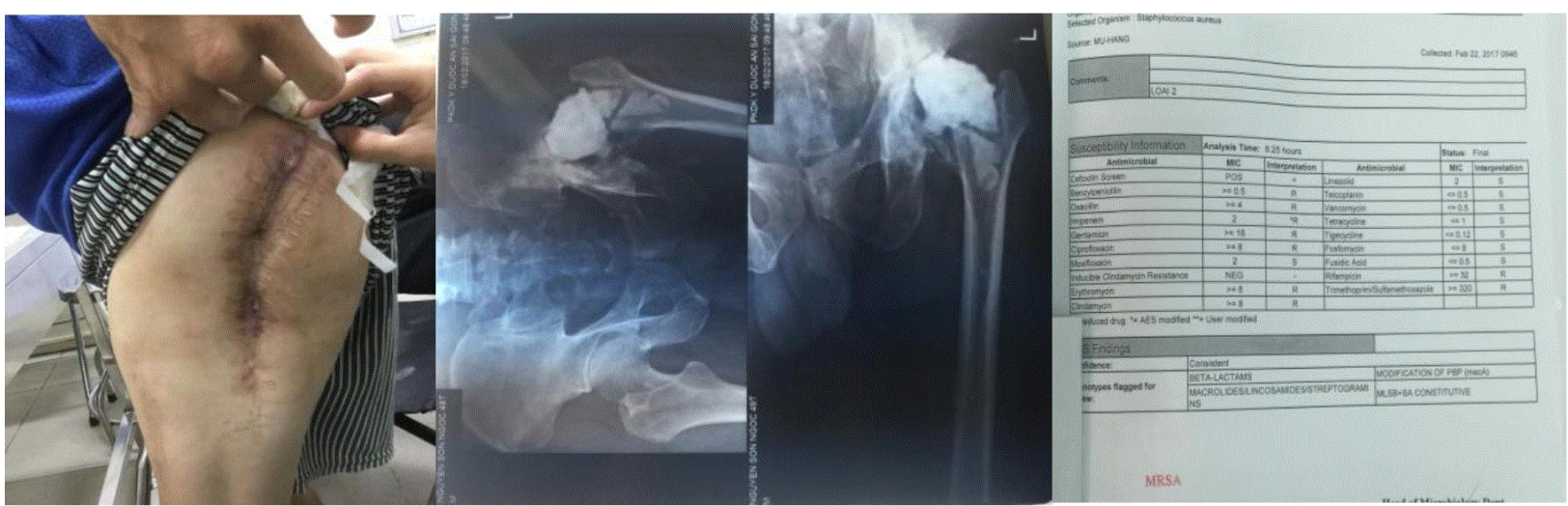

Figure 7: Before operation.

His results after 1 month of follow-up: healing wound, normal WBC results, ESR: $1 \mathrm{~h}: 22 \mathrm{~mm}, 2 \mathrm{~h} 47 \mathrm{~mm}$. CRP: $11.2 \mathrm{mg} / \mathrm{L}$.

After 3 months, he checked up in Cho Ray hospital, his wound was healed, left leg function was good, and infectious condition was improved. Serological Tests: WBC: normal, ESR: normal, CRP: 2,9 $\mathrm{mg} / \mathrm{L}$ (Figure 8).

He also underwent left hip revision arthroplasty after 6 months.

\section{Discussion}

To use an articulating, antibiotic-loaded PMMA cement spacer is a simple and fast molding method to fit all defects and allows early mobilization and efficient local antibiotic delivery $[9,10]$.

PJI hard to treat, but results reported in the literature have shown improvement over time up to a cure rate of $90 \%[11,12]$.

In 2007, Masri et al. followed up 29 patients for an average of 47 months and found an infection recurrence rate of $10.3 \%$ [13]. Durbhakula et al. reported a $100 \%$ success rate in 20 patients followed up for an average of 38 months in 2004 [14].
Several major questions regarding two-stage revision remain unanswered, such as the optimal du-ration of intravenous antibiotic use and whether oral antibiotics should be used during the interim period and after final reconstruction, or the kind and amount of antibiotic to impregnate with cement. We chose the kind of antibiotics, such as heat-stable, powdered form and broad spectrum, and depended on antibiogramme.

Our results showed clearly the improved function between stages and we believe that a mobile hip allows easier second-stage operation and facilitates rehabilitation.

The commercially available molding system is the StageOne Hip Cement Spacer Molds (Biomet Orthopedics Inc., Warsaw, IN). This produces significantly better congruency of the articulating parts, with better stability. Because the molds are made of silicone, the surface of the spacers is not compressed during the hardening process. Also, these molds are only for single use, which is quite costly. We have developed a tapered aluminium mold for production of a custommade PMMA spacer during operation. The mold can be cleaned and sterilized for reuse. 
Citation: Hung TNK, Duong TB, Tu DT, Nhan LNT, Thi C, et al. (2018) Prosthetic Hip Joint Infection: An Aluminium Mold for Intraoperative Production of Antibiotic-loaded Cement Hip Prostheses: 3 Cases Report. Med Rep Case Stud 3: 153. doi:10.4172/2572-5130.1000153

Page 5 of 5

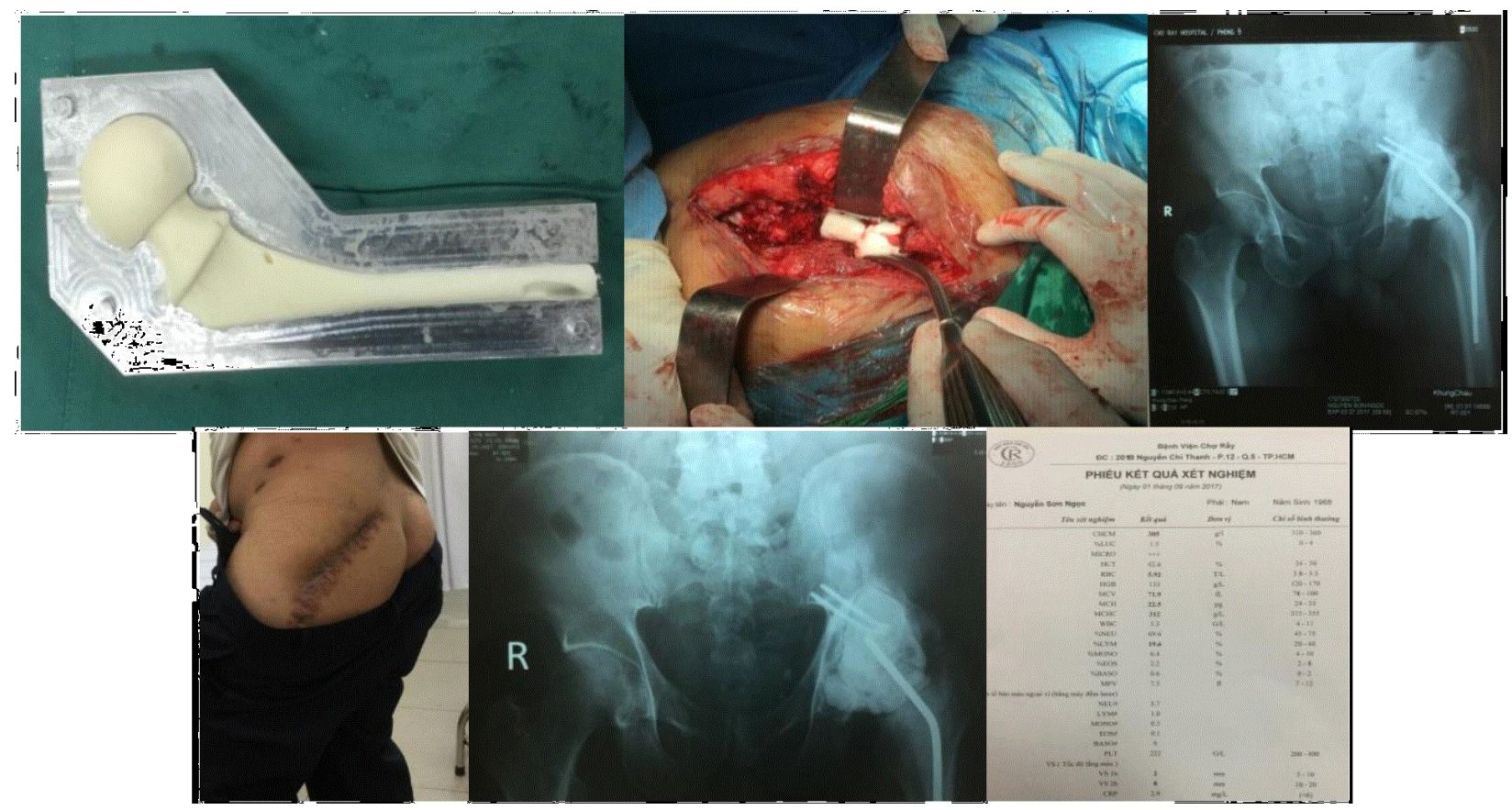

Figure 8: After 3 months.

\section{Conclusion}

The two stage revision hip arthroplasty protocol offers the greatest chance for eradication of infection. Although it has been the gold standard for the treatment of patients with chronic PJI, treating hip prosthetic infection with these articulating spacers eradicates infection effectively, improves the life quality before reimplantation and provides good final results without significant complications.

\section{Conflict of Interests}

The authors declare that there is no conflict of interests regarding the publication of this paper.

\section{References}

1. Kurtz SM, Lau E, Watson H, Schmier JK, Parvizi J (2012) Economic burden of periprosthetic joint infection in the United States. J Arthroplasty 27: 61-65.e1.

2. Berend KR, Lombardi Jr AV, Morris MJ, Bergeson AG, Adams JB, et al. (2013) Two-stage treatment of hip periprosthetic joint infection is associated with a high rate of infection control but high mortality. Clin Orthop Relat Res 471: 510-518.

3. Bozic KJ, Ries MD (2005) The impact of infection after total hip arthroplasty on hospital and surgeon resource utilization. J Bone Joint Surg Am 87: 1746-1751.

4. Kurtz SM, Lau E, Schmier J, Ong KL, Zhoa K, et al. (2008) Infection burden for hip and knee arthroplasty in the United States. J Arthroplasty 23: 984-991.

5. Anderson DJ, Sexton DJ, Kanafani ZA, Auten G, Kaye KS (2007) Severe surgical site infection in community hospitals: epidemiology, key pro- cedures, and the changing prevalence of methicillin-resistant Staphylococcus aureus. Infect Control Hosp Epidemiol 28: 1047-1053.

6. Parvizi J, Ghanem E, Azzam K, Davis E, Jaberi F, et al. (2008) Periprosthetic infection: are current treatment strategies adequate? Acta or-thopaedica Belgica 74: 793-800.

7. Bradbury T, Fehring TK, Taunton M, Hanssen A, Azzam K, et al. (2009) The fate of acute methicillin-resistant Staphylococcus aureus periprosthetic knee infections treated by open debridement and retention of components. J Arthroplasty 24: 101-104.

8. Parvizi J, Azzam K, Ghanem E, Austin MS, Rothman RH (2009 Periprosthetic infection due to resistant staphylococci: serious problems on the horizon. Clin Orthop Relat Res 467: 1732-1739.

9. Pearle AD, Sculco TP (2002) Technique for fabrication of an antibioticloaded cement hemiarthro-plasty (ANTILOCH) prosthesis for infected total hip arthroplasty. Am J Orthop (Belle Mead NJ) 31: 425-427.

10. Etienne G, Waldman B, Rajadhyaksha AD, Ragland PS, Mont MA (2003) Use of a functional temporary prosthesis in a two-stage approach to infection at the site of a total hip arthroplasty. J Bone Joint Surg Am 85-A: 94-96.

11. Wang JW, Chen CE (1997) Reimplantation of infected hip arthroplasties using bone allografts. Clin Orthop Relat Res 335: 202-210.

12. Kilgus DJ, Howe DJ, Strang A (2002) Results of periprosthetic hip and knee infections caused by resistant bacteria. Clin Orthop Relat Res 404: 116-124.

13. Masri BA, Panagiotopoulos KP, Greidanus NV, Garbuz DS, Duncan CP (2007) Cementless Two-Stage Exchange Arthroplasty for Infection after Total Hip Arthroplasty. The Journal of Arthroplasty 22: 72-78.

14. Durbhakula SM, Czajka J, Fuchs MD, Uhl RL (2004) Spacer endoprosthesis for the treatment of infected total hip arthro-plasty. The Journal of Arthroplasty 19: 760-767. 